\title{
ATAD2 expression increases [18F] Fluorodeoxyglucose uptake value in lung adenocarcinoma via AKT-GLUT1/HK2 pathway
}

\author{
Tong Sun, Bulin Du, Yao Diao, Xuena Li, Song Chen \& Yaming Li* \\ Department of Nuclear Medicine, The first Hospital of China Medical University, Liaoning 110001, China
}

\begin{abstract}
[18F]Fluorodeoxyglucose (FDG) PET/CT imaging has been widely used in the diagnosis of malignant tumors. ATPase family AAA domain-containing protein 2 (ATAD2) plays important roles in tumor growth, invasion and metastasis. However, the relationship between [18F]FDG accumulation and ATAD2 expression remains largely unknown. This study aimed to investigate the correlation between ATAD2 expression and [18F]FDG uptake in lung adenocarcinoma (LUAD), and elucidate its underlying molecular mechanisms. The results showed that ATAD2 expression was positively correlated with maximum standardized uptake value $\left(S_{U} V_{\text {max }}\right)$, total lesion glycolysis (TLG), glucose transporter type 1 (GLUT1) expression and hexokinase2 (HK2) expression in LUAD tissues. In addition, ATAD2 knockdown significantly inhibited the proliferation, tumorigenicity, migration, [18F]FDG uptake and lactate production of LUAD cells, while, ATAD2 overexpression exhibited the opposite effects. Furthermore, ATAD2 modulated the glycometabolism of LUAD via AKT-GLUT1/HK2 pathway, as assessed using LY294002 (an inhibitor of PI3K/AKT pathway). In summary, to explore the correlation between ATAD2 expression and glycometabolism is expected to bring good news for anti-energy metabolism therapy of cancers. [BMB Reports 2019; 52(7): 457-462]
\end{abstract}

\section{INTRODUCTION}

Lung cancer is one of the most common malignancy worldwide, with rising incidence and mortality rates yearly (1). Adenocarcinoma is a histological subtype of non-small cell lung cancer, which is commonly treated by surgery, chemotherapy and targeted therapies (2). Rapid tumor progression, prone to metastasis and chemotherapy resistance have emerged as the

*Corresponding author. Tel: +86-24-83282142; Fax: +86-24-83282 671; E-mail: ymli2001@163.com

https://doi.org/10.5483/BMBRep.2019.52.7.042

Received 11 February 2019, Revised 13 March 2019, Accepted 13 May 2019

Keywords: ATAD2, GLUT1, HK2, Lung adenocarcinoma, [18F]FDG accumulation challenges in cancer treatment. Hence, the discovery of molecular markers reflecting tumor's biological behavior can be of great value for targeting anticancer drug resistance. ATPase family AAA domain-containing protein 2 (ATAD2) is composed of an ATPase domain and a bromodomain that located on human chromosome $8 q 24$, with possible occurrence of nuclear chromatin acetylation $(3,4)$. ATAD2 is involved in several regulatory mechanisms, such as cell proliferation and metastasis, and holds great promise as a new prognostic marker $(5,6)$.

Fluorodeoxyglucose (FDG) is a structural analogue of glucose, and [18F]FDG is the most widely used in PET imaging for cancer diagnosis. [18F]FDG uptake is increased in most malignant tumors, and can be associated with the overexpression of glucose transporter type 1 (GLUT1) and hexokinase 2 (HK2) (7). Tumor cells are notoriously dependent on glucose metabolism as the primary energy source for adenosine triphosphate (ATP) synthesis. ATAD2 is a member of AAA + ATPase family (8), which plays important roles in ATPase activity and ATP production. However, the relationship between ATAD2 expression and glycometabolism remains largely unclear. The present study aimed to investigate the correlation between ATAD2 expression and [18F]FDG uptake in lung adenocarcinoma (LUAD). In addition, the potential underlying molecular mechanisms were elucidated by detecting the expression levels of GLUT1 and HK2 in LUAD after ATAD2 transfection.

\section{RESULTS}

ATAD2 expression is up-regulated in LUAD tissues and cell lines

To investigate the role of ATAD2 in LUAD, ATAD2 expression levels were detected in 66 LUAD tissues and 52 adjacent normal lung tissues. As shown in Fig. 1A, the expression levels of ATAD2 were up-regulated in LUAD tissues compared to normal tissues. The positive expression rates of ATAD2 were $72.7 \%(48 / 66)$ and $19.2 \%(10 / 52)$ in LUAD tissues $(n=66)$ and adjacent normal lung tissues ( $\mathrm{n}=52)$, respectively. Besides, it was found that the expression levels of ATAD2 were higher in LUAD cell lines compared to HBE cell line, as revealed by qRT-PCR and Western blot analyses (Fig. 1B). Table 1 shows the correlation between ATAD2 expression levels and clinicopathological characteristics of patients with LUAD. Statistical analysis revealed that ATAD2 overexpression 
A

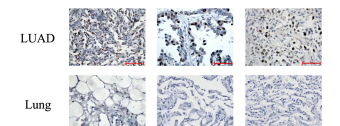

B

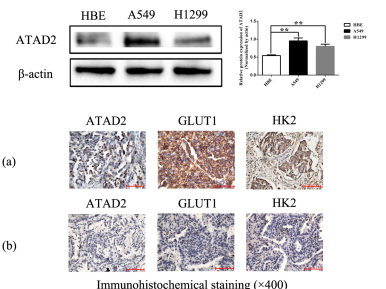

(c)
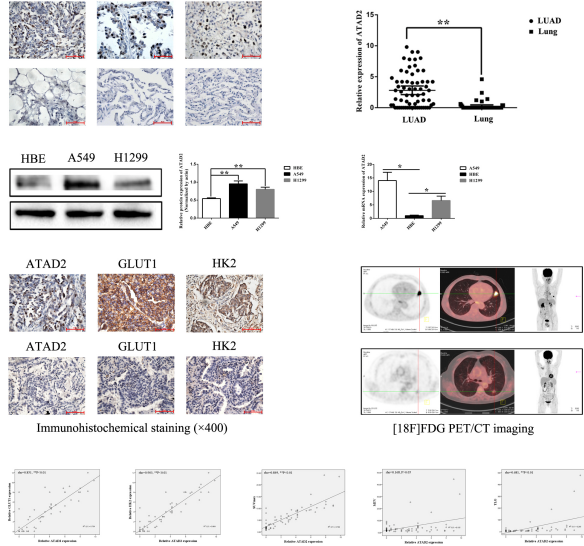

Fig. 1. Up-regulation of ATAD2 expression in LUAD and the relationship between ATAD2 expression and glycometabolism. (A) Immunohistochemical staining of ATAD2 expression in LUAD tissues and adjacent normal lung tissues $(\times 400)$. The expression level of ATAD2 was increased in LUAD tissues compared to normal lung tissues (Mann-Whitney $U$ test; $Z=-6.236, * P<0.01$ ). (B) The protein and mRNA expression levels of ATAD2 were up-regulated in LUAD cell lines, as revealed by Western blot and qRT-PCR analyses respectively (two-tailed Student's t-test; $* \mathrm{P}<0.05, * * \mathrm{P}<0.01$ ). (C) Correlation of ATAD2 expression with [18F]FDG accumulation and the expression levels of GLUT1 and HK2. (a) A 58-year-old male patient with left LUAD exhibited high ATAD2 expression. [18F]FDG PET/CT scans showed intense [18F]FDG accumulation in LUAD tissues $\left(\mathrm{SUV}_{\max }=10.98 ; \mathrm{MTV}=1.4 ; \quad \mathrm{TLG}=10.89\right)$. Immunohistochemical analysis revealed high expression levels of GLUT1 and HK2 $(\times 400)$. (b) A 58-year-old female patient with left LUAD exhibited low ATAD2 expression. [18F]FDG PET/CT scans demonstrated modest [18F]FDG accumulation in LUAD tissues $\left(\mathrm{SUV}_{\max }=1.49 ; \mathrm{MTV}=1.83 ; \mathrm{TLG}=1.79\right)$. Immunohistochemical analysis revealed low expression levels of GLUT1 and HK2 $(\times 400)$. (c) The expression levels of ATAD2 were positively correlated with SUV $_{\text {max }}$, TLG, GLUT1 expression and HK2 expression $(* * P<0.01$ ), but not MTV (P $>0.05)$ by Spearman's test. Bars in immunohistochemical images represent $50 \mu \mathrm{m}$.

was closely related to the positive lymphatic metastasis and poor differentiation, but not significantly correlated with age, gender and tumor size. Moreover, the high expression levels of ATAD2 significantly predicted poor LUAD progression compared to low ATAD2 expression by analyzing The Cancer Genome Atlas (TCGA) database (http://tumorsurvival.org/ index.html) (Supplementary Fig. S1A). These results suggest that ATAD2 may promote oncogenesis in LUAD patients.

\section{ATAD2 expression is positively correlated with $S U V_{\max }, T L G$,} GLUT1 expression and HK2 expression

To examine the correlation between ATAD2 expression and semi-quantitative indices of [18F]FDG PET/CT imaging and glycometabolism, maximum standardized uptake value $\left(\mathrm{SUV}_{\max }\right)$, metabolic tumor volume (MTV) and total lesion glycolysis (TLG) were measured, and the expression levels of ATAD2, GLUT1 and HK2 in 66 LUAD tissues were determined by immunohistochemical staining. Notably, the expression levels of ATAD2 in LUAD were positively correlated with SUV $_{\max }$ and TLG, but not MTV (Fig. 1C). SUV max $_{\max }$ was positively correlated with tumor stage (Supplementary Table S1). In addition, ATAD2 expression was significantly correlated with the expression levels of GLUT1 and HK2 in LUAD tissues (Fig. 1C). These results indicate that ATAD2 may promote [18F]FDG uptake and glycometabolism in LUAD.

\section{ATAD2 promotes proliferation, tumorigenicity and migration of LUAD cells}

To determine whether ATAD2 can affect the proliferation, tumorigenicity and migration of LUAD cells, both A549 and H1299 cells were transfected with ATAD2 siRNAs and plasmid. As shown in Fig. $2 \mathrm{~A}$ and $2 \mathrm{~B}$, the siRNAs were effective at silencing ATAD2 expression, while the plasmid was effective at overexpressing ATAD2, as confirmed by qRT-PCR and Western blot analyses. Cell proliferation, tumorigenicity and cell migration capacity were evaluated by $\mathrm{EdU}$, colony formation and transwell assays, respectively. The results demonstrated that ATAD2 knockdown significantly inhibited the proliferation, tumorigenicity and migration of A549 and H1299 cells (Fig. 2C, $2 \mathrm{E}$ and $2 \mathrm{G}$, respectively). In contrast, ATAD2 overexpression enhanced the proliferation, tumorigenicity and migration capacity of A549 and H1299 cells (Fig. 2D, 2F and 2H, respectively). PI3K/AKT signaling pathway plays essential roles in the proliferation and migration of tumor cells. Furtherly, after LY294002 (an inhibitor of PI3K/AKT pathway; $20 \mu \mathrm{M}$ ) treatment, the results indicated that ATAD2 promoted cell proliferation, tumorigenicity and migration via PI3K/AKT pathway (Fig. 2D, 2F and $2 \mathrm{H}$, respectively).

\section{ATAD2 promotes glycometabolism in LUAD cells}

To elucidate the role of ATAD2 in glycometabolism, the expression levels of GLUT1 and HK2 were examined by Western blot analysis. The results showed that the protein expression levels of GLUT1 and HK2 were significantly decreased in both A549 and H1299 cells following ATAD2 down-regulation (Fig. $3 \mathrm{~A})$, whereas the increased expression levels of GLUT1 and HK2 were observed ATAD2 up-regulation (Fig. 3B). Cellular glucose uptake, lactic acid production and adenosine triphosphate (ATP) level were determined to assess the effects of ATAD2 in glucose metabolism. [18F]FDG uptake was used to evaluate the glucose uptake capacity in LUAD cells. Glucose-containing or glucose-free medium was replaced $2 \mathrm{~h}$ before the addition of [18F]FDG. As shown in Fig. 3C and 3D, [18F]FDG uptake was decreased after ATAD2-siRNAs transfection and increased after ATAD2-plasmid transfection in both A549 and H1299 cells incubated with or without glucose. To further explore the end-products of glycometabolism, lactic acid and ATP levels were determined. The results showed that lactic acid production were reduced in LUAD cells transfected with siRNAs, while elevated in LUAD cells following ATAD2-plasmid transfection (Fig. 3E and 3F). However, there were no significant changes in 
Table 1. Correlation between ATAD2 expression and the clinicopathological characteristics of patients with lung adenocarcinoma by Mann-Whitney $\mathrm{U}$ or Kruskal-Wallis $\mathrm{H}$ tests

\begin{tabular}{|c|c|c|c|c|}
\hline Clinicopathological characteristics & No. of patients & ATAD2 expression level (mean \pm SD) & $\mathrm{Z} \chi^{2}$ & Pvalue \\
\hline Age (year) & & & -0.182 & 0.856 \\
\hline$\leq 60$ & 34 & $2.81 \pm 2.916$ & & \\
\hline$>60$ & 32 & $2.81 \pm 2.767$ & & \\
\hline Gender & & & -0.019 & 0.984 \\
\hline Male & 31 & $2.93 \pm 3.108$ & & \\
\hline Female & 35 & $2.70 \pm 2.586$ & & \\
\hline Tumor size $(\mathrm{cm})$ & & & -1.523 & 0.128 \\
\hline$\leq 2$ & 27 & $2.08 \pm 2.272$ & & \\
\hline$>2$ & 39 & $3.31 \pm 3.076$ & & \\
\hline Lymphatic metastasis & & & -3.264 & $0.001 * *$ \\
\hline Negative & 47 & $2.06 \pm 2.434$ & & \\
\hline Positive & 19 & $4.67 \pm 2.911$ & & \\
\hline Tumor differentiation grade & & & 9.484 & $0.009 * *$ \\
\hline Well & 20 & $1.34 \pm 1.975$ & & \\
\hline Moderately & 33 & $2.81 \pm 2.356$ & & \\
\hline Poorly & 13 & $5.08 \pm 3.608$ & & \\
\hline
\end{tabular}

$* * \mathrm{P}<0.01$

ATP content whether ATAD2 expression level was up- or down-regulated (Fig. $3 \mathrm{G}$ and $3 \mathrm{H}$ ). These findings indicate that ATAD2 can promote the glycometabolism of LUAD, possibly through the mechanisms of GLUT1 and HK2 up-regulation.

\section{ATAD2 promotes glycometabolism in LUAD via AKT-GLUT1/HK2 pathway}

AKT is known to play an important role during the activation of GLUT1 and HK2. Therefore, the expression levels of AKT and phosphorylated AKT (pAKT) were measured in LUAD cells transfected with ATAD2-siRNAs and ATAD2-plasmid. The expression levels of pAKT were significantly reduced after ATAD2-siRNAs transfection and increased after ATAD2-plasmid transfection in both A549 and H1299 cells (Fig. 3A and 3B). Furtherly, the results showed that the expression levels of GLUT1, HK2 and pAKT were significantly decreased in A549 and H1299 cells treated with $20 \mu \mathrm{M}$ LY294002 (Fig. 3B). Likewise, [18F]FDG uptake (Fig. 3D) and lactate production (Fig. 3F) were reduced after LY294002 treatment. However, no significant change in ATP level was observed in LUAD cells (Fig. $3 \mathrm{H})$. Taken together, the above findings suggest that ATAD2 may regulate glycometabolism in LUAD via AKT-GLUT1/HK2 pathway.

\section{DISCUSSION}

The expression of ATAD2 in various malignant tumors is significantly higher than that in normal tissues (9), and ATAD2 has been used to evaluate the clinical prognosis of various cancers, such as lung cancer (10, 11), breast cancer (11), hepatocellular carcinoma (12), ovarian cancer (13), endometrial cancer (14) and gastric cancer (15), which are consistent with the results of ATAD2 expression in our study. Notably, we found that the expression levels of ATAD2 were up-regulated in LUAD tissues and cell lines compared to normal lung tissues and cell lines, respectively (Fig. 1A and 1B). Furthermore, we confirmed that ATAD2 overexpression was closely related to the positive lymphatic metastasis and poor tumor differentiation (Table 1), as well as poor LUAD progression by analyzing TCGA database (Supplementary Fig. S1A). However, the survival analysis revealed that there was no significant difference in LUAD patient survival time between ATAD2 high-expression group $(\mathrm{n}=33)$ and ATAD2 low-expression group $(\mathrm{n}=33$ ) via the Kaplan-Meier method (Supplementary Fig. S1B). This probably due to the short follow-up period of our cases, and we will continue to follow them in future work.

[18F]FDG PET/CT is the most widely used in vivo molecular imaging technique (16). Semi-quantitative indicators, such as SUV $_{\max }$, MTV and TLG, can reflect the state of glycometabolism in tumor cells. In this study, for the first time, we found that ATAD2 expression was positively correlated with $S U V_{\max }, T L G$, GLUT1 expression and HK2 expression in LUAD (Fig. 1C). Previous studies have shown that one of the reasons for increasing glycometabolism in malignant tumor cells is the overexpression of glucose transporters, especially GLUT1 and GLUT3. High expression of GLUT1 is predominantly found in LUAD (17). Besides, hexokinase is a rate-limiting enzyme that catalyzes the initial step of cellular glycometabolism (18). In most malignant tumors, HK2 is highly expressed and plays a crucial role in the regulation of high-rate glycolysis $(19,20)$. Both GLUT1 and HK2 can be used to reflect the alterations of 


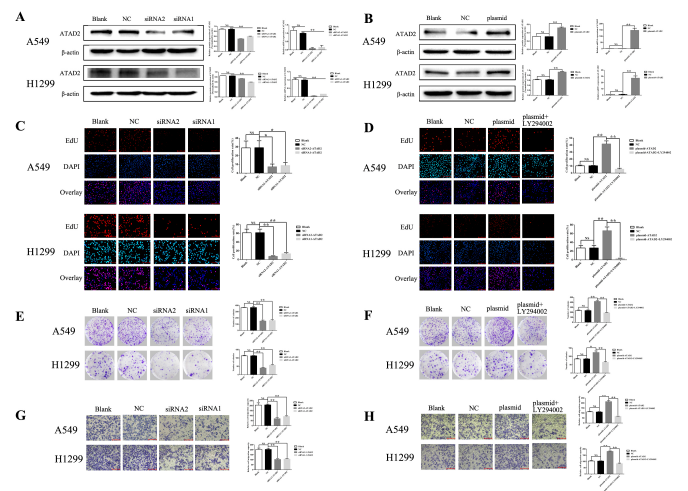

Fig. 2. ATAD2 promotes the proliferation, tumorigenicity and migration of LUAD cells. (A) The mRNA and protein expression levels of ATAD2 were down-regulated after transfected with siRNAs. (B) The mRNA and protein expression levels of ATAD2 were up-regulated after transfected with plasmid. (C) ATAD2 knockdown decreased cell proliferation, as evaluated by EdU assay. (D) Cell proliferation after ATAD2 up-regulation and LY294002 treatment was assessed using EdU assay. (E) ATAD2 knockdown decreased tumorigenicity, as revealed by colony formation analysis. (F) Assessment of tumorigenicity was performed by colony formation analysis after ATAD2 up-regulation and LY294002 treatment. (G) ATAD2 knockdown reduced cell migration, as shown by transwell assay. $(\mathrm{H})$ Evaluation of cell migration capacity was performed using transwell assay after ATAD2 up-regulation and LY294002 treatment (two-tailed Student's t-test, ${ }^{*} \mathrm{P}<0.05,{ }^{*} \mathrm{P}<0.01 ; \mathrm{NC}=$ negative control, $\mathrm{NS}=$ not significant; magnification: $\times 200$, bars in EdU and transwell images represent $100 \mu \mathrm{m})$.

glycometabolism in LUAD. Therefore, it is believed that ATAD2 can promote glycometabolism by overexpressing GLUT1 and HK2 in LUAD.

ATAD2 is an essential determinant of tumor proliferation and metastasis $(6,21)$. In the present study, we confirmed that ATAD2 induced cell proliferation (Fig. 2C, D), colony formation ability (Fig. 2E, F) and migration capacity (Fig. 2G, H) in vitro, which were consistent with previous findings. To investigate the effects of ATAD2 on glycometabolism, the protein expression levels of GLUT1 and HK2 were determined, as well as cellular glucose uptake, lactic acid production and ATP level. The results showed that the levels of GLUT1 expression, HK2 expression, [18F]FDG uptake and lactic acid were significantly decreased in both A549 and H1299 cells after silencing ATAD2 (Fig. 3A, C, E), while overexpressing ATAD2 exhibited the opposite effects (Fig. 3B, D, F). These findings support that ATAD2 can promote glycometabolism and increase the production of metabolic products. However, ATP level was not significantly altered whether ATAD2 overexpressed or silenced (Fig. 3G, H). This is probably related to the compensatory allocation of energy in tumor cells. Furthermore, ATAD2 is a member of ATPase family that catalyzes the hydrolysis of ATP into ADP, and the ATP produced by glycometabolism may be counteracted. The underlying cause remains to be determined in future studies.

$\mathrm{PI} 3 \mathrm{~K} / \mathrm{AKT}$ pathway plays an important role in the glycometa-

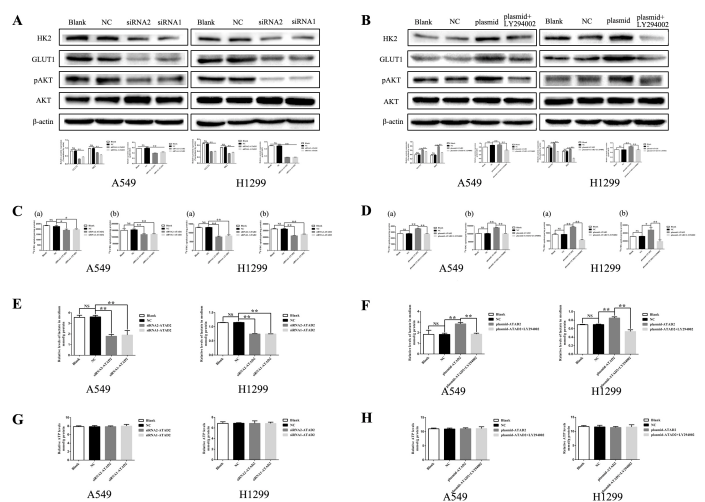

Fig. 3. ATAD2 promotes glycometabolism in LUAD cells via AKT-GLUT1/HK2 pathway. (A) The relative protein expression levels of target proteins were determined after ATAD2 knockdown. (B) The relative protein expression levels of target proteins were determined after ATAD2 up-regulation and LY294002 treatment. (C) [18F]FDG uptake was suppressed with (a) or without (b) glucose after ATAD2 knockdown. (D) Effects of ATAD2 overexpression and LY294002 on [18F]FDG uptake incubated with (a) or without (b) glucose. (E) Effect of ATAD2 siRNAs on cellular lactate production. (F) Effects of ATAD2 overexpression and LY294002 on cellular lactate production. (G) Effect of ATAD2 siRNAs on cellular ATP content. $(\mathrm{H})$ Effects of ATAD2 overexpression and LY294002 on cellular ATP content (two-tailed Student's t-test, $* \mathrm{P}<0.05, * * \mathrm{P}<0.01 ; \mathrm{NC}=$ negative control, NS = not significant).

bolic reprogramming of tumor cells. AKT, as known as Warburg kinase, is a core factor of PI3K/AKT pathway (22). The activation of Myc in tumors continuously activates PI3K/AKT pathway and induces AKT downstream transcription factors for the regulation of tumor glycometabolism (23). Oncogenic activation of the PI3K/AKT pathway could promote cellular glucose uptake (24). Myc induces metabolic reprogramming, involving glycolysis, which is necessary for efficient cell proliferation $(25,26)$. Ciro et al. (27) have reported that ATAD2 is a co-activator of Myc, which can enhance its transcriptional activity. Fan et al. (10) have shown that the activation of Myc promotes cellular glucose uptake, glycolysis and lactic acid production. Our results revealed that the expression levels of PAKT were decreased in both A549 and H1299 cells after ATAD2 knockdown (Fig. 3A), and these effects were opposed by ATAD2 overexpression (Fig. 3B). In addition, it was noted that the expression levels of GLUT1, HK2 and pAKT (Fig. 3B), as well as [18F]FDG uptake (Fig. 3D) and lactic acid production (Fig. 3F) were reduced by LY294002 treatment. Overall, ATAD2 co-activates Myc, induces the activation of PI3K/AKT pathway, and promotes the expression levels of GLUT1 and HK2, thus leading to an increase in glycometabolism rate (Supplementary Fig. S2).

In summary, ATAD2 exhibits a significant positive correlation with glycometabolism in LUAD, regulates cell proliferation, tumorigenicity and migration capacity, as well as promotes [18F]FDG uptake and lactate production via AKT-GLUT1/HK2 pathway. To explore the correlation between ATAD2 expression 
and glycometabolism is expected to bring good news for anti-energy metabolism therapy of cancers.

\section{MATERIALS AND METHODS}

\section{Study population}

Sixty-six patients (31 men and 35 women; mean age, $59.91 \pm$ 9.79 years) with LUAD were included in this study. All of them underwent [18F]FDG PET/CT scan before tumor resection at the First Hospital of China Medical University from June 2015 to August 2016. The inclusion criteria were as follows: i) the diagnosis of LUAD was confirmed by pathologic examination of the surgical specimens $(n=66)$; and ii) the surgical excision specimens containing normal lung tissues $(n=52)$ were used as a control group. Patients who i) received chemotherapy/radiotherapy before PET/CT scanning, and ii) suffered from multiple cancers were excluded from this study. Demographic and clinical characteristics were recorded and analyzed. This study was approved by the ethics committee of the First Hospital of China Medical University (IRB number: AF-SOP-07-1. 1-01), and all participants signed a written informed consent form.

\section{PET/CT imaging}

PET/CT imaging and data acquisition were performed using an integrated PET/CT scanner (Siemens, Germany). For quantitative analysis, the volume of interest (VOI) was delineated, while $\mathrm{SUV}_{\max }$, average standardized uptake value $\left(\mathrm{SUV}_{\text {mean }}\right)$ and MTV were measured. VOI was delineated automatically, and the threshold of SUV mean $_{\text {and }}$ MTV was 50\%. TLG was calculated using the following formula: TLG $=\mathrm{SUV}_{\text {mean }} \times \mathrm{MTV}$. Each index was measured three times to obtain an average value.

\section{Immunohistochemistry}

Five micrometer sections of formalin-fixed and paraffinembedded LUAD tissues $(n=66)$ and normal lung tissues $(n=$ 52) were immunohistochemically stained with anti-ATAD2 antibodies using EliVision two-step staining method. Meanwhile, the 66 LUAD tissue samples were incubated with anti-GLUT1 and anti-HK2 antibodies. All antibodies were purchased from Abcam (UK). The color strengths of immuohistochemical analysis were defined as follows: 0 (colorless), 1 (yellow), 2 (light brown) and 3 (dark brown). The number of positive cells per field of vision was measured and classified as 0 (none), 1 (1-10\%), 2 $(11-50 \%), 3(51-80 \%)$ and 4 (> $80 \%)$. The score of one visual field was obtained by multiplying the above two scores, and the total score was the mean of the five visual field scores.

\section{Cell culture and transfection}

Human LUAD cell lines (A549 and H1299) and human bronchial epithelial (HBE) cell line were obtained from the Chinese Academy of Sciences. A549 cells were grown in Dulbecco modified Eagle medium (DMEM; HyClone, USA), while $\mathrm{H} 1299$ and $\mathrm{HBE}$ cells were grown in RPMI-1640 medium (HyClone, USA) supplemented with 10\% fetal bovine serum
(FBS; CLACK, USA) at $37^{\circ} \mathrm{C}$ and $5 \% \mathrm{CO}_{2}$. Lipofectamine 3000 (Invitrogen, USA) was used for transient transfection, according to the manufacturer's protocol. ATAD2-siRNAs and control whose targeting sequences used in this study were listed in Supplementary Table S2. ATAD2-siRNAs and control were purchased from Pharma (Viewsolid, China). ATAD2 overexpressing cell lines were transfected with ATAD2_RC218291 plasmid; while pCMV6-Entry (PS100001; Origene, USA) was used as a negative control plasmid. In addition, LY294002 (Abcam, UK) was used to inhibit PI3K/AKT pathway.

\section{Cell proliferation, tumorigenicity and migration assays}

Cell proliferation was evaluated using YF594 Click-iT EdU Imaging Kit (Everbright, USA). After EdU incubation, cells were fixed and stained with Click-iT reaction mixture according to the manufacturer's protocol. Moreover, cell nuclei were stained with Hoechst33342. The captured images were used to calculate the percentage of EdU-positive cells via Image J software. For colony formation assay, the cells were seeded into 6-well plates at a density of 500 cells per well. After culturing in the cell incubator $\left(37^{\circ} \mathrm{C}, 5 \% \mathrm{CO}_{2}\right)$ for 14 days, the cells were fixed in $70 \%$ ethanol and then stained by crystal violet. The migration capacity of cells was examined by a transwell assay. Briefly, $2 \times$ $10^{5}$ cells resuspended in $200 \mu \mathrm{l}$ serum-free medium were transferred into the upper chamber; while $600 \mu \mathrm{l}$ of medium containing $10 \%$ FBS was loaded into the lower chamber. After 14 $\mathrm{h}$ of incubation, the cells passed through the membrane were stained with $0.1 \%$ crystal violet. Finally, the images were recorded and analyzed. Each assay was repeated for three times.

\section{Cell metabolism assay}

To examine the changes in cell metabolism, cellular ATP level, glucose uptake and lactic acid production were determined. Relative cellular ATP content was measured using an ATP assay kit (Beyotime, China) according to the manufacturer's instructions. [18F]FDG cellular uptake can be used to evaluate the capacity of glucose uptake in tumor cells. [18F]FDG at a final concentration of $4 \mu \mathrm{Ci} / \mathrm{ml}$ was added and cultured at $37^{\circ} \mathrm{C}$ and $5 \% \mathrm{CO}_{2}$ for 60 min. Cell lysates were prepared using trypsin, and the radioactivity of the whole-cell lysates was then assessed using gamma counter. The levels of lactate in the external medium were measured using a lactic acid assay kit (KeyGEN, China) according to the manufacturer's instructions. These readouts were normalized to the amount of the corresponding protein. All experiments were carried out independently in triplicate.

\section{Quantitative real-time polymerase chain reaction (qRT-PCR)} and westem blot analyses

Please see the supplementary information.

\section{Statistical analysis}

All statistical analyses were performed using SPSS (version 22.0). Statistical differences between two or three groups were compared by Mann-Whitney U, Kruskal-Wallis $\mathrm{H}$ and two-tailed 
Student's $t$-tests; while Spearman's test was used for correlation analysis. Survival analysis of LUAD patients were carried out using the Kaplan-Meier method. Data were presented as mean \pm SD. $P$ values of less than 0.05 were considered statistically significant $\left({ }^{*} \mathrm{P}<0.05\right.$ and $\left.{ }^{* * P}<0.01\right)$. Figures were plotted using GraphPad Prism (version 6.0).

\section{ACKNOWLEDGEMENTS}

This study was supported by Natural Science Foundation of China (No. 81771868).

\section{CONFLICTS OF INTEREST}

The authors have no conflicting interests.

\section{REFERENCES}

1. Siegel RL, Miller KD and Jemal A (2018) Cancer statistics, 2018. CA Cancer J Clin 68, 277-300

2. Coudray N, Moreira AL, Sakellaropoulos T, Fenyo D, Razavian N and Tsirigos A (2018) Classification and mutation prediction from non-small cell lung cancer histopathology images using deep learning. Nat Med 24, 1559-1567

3. Yamasaki $T$, Nakazaki $Y$, Yoshida $M$ and Watanabe $Y$ (2011) Roles of conserved arginines in ATP-binding domains of $\mathrm{AAA}+$ chaperone $\mathrm{ClpB}$ from Thermus thermophiles. FEBS J 278, 2395-2403

4. Nouet C, Truan G, Mathieu L and Dujardin G (2009) Functional analysis of yeast bcs 1 mutants highlights the role of Bcs1p-specific amino acids in the AAA domain. J Mol Biol 388, 252-261

5. Zheng L, Li T, Zhang Y et al (2015) Oncogene ATAD2 promotes cell proliferation, invasion and migration in cervical cancer. Oncol Rep 33, 2337-2344

6. Fouret R, Laffaire J, Hofman P et al (2012) A comparative and integrative approach identifies ATPase family, AAA domain containing 2 as a likely driver of cell proliferation in lung adenocarcinoma. Clin Cancer Res 18, 5606-5616

7. Alvarez JV, Belka GK, Pan T et al (2014) Oncogene pathway activation in mammary tumors dictates [18F]-FDG-PET uptake. Cancer Res 74, 7583-7598

8. Zou JX, Revenko AS, Li LB, Gemo AT and Chen HW (2007) ANCCA, an estrogen-regulated AAA + ATPase coactivator for ERalpha, is required for coregulator occupancy and chromatin modification. Proc Natl Acad Sci U S A 104, 18067-18072

9. Zhang $Y$, Sun $Y$, Li $Y$ et al (2013) ANCCA protein expression is a novel independent poor prognostic marker in surgically resected lung adenocarcinoma. Ann Surg Oncol 20, 577-582

10. Fan Y, Dickman KG, Zong WX (2010) Akt and c-Myc differentially activate cellular metabolic programs and prime cells to bioenergetic inhibition. J Biol Chem 285, 7324-7333

11. Caron C, Lestrat C, Marsal S et al (2010) Functional characterization of ATAD2 as a new cancer/testis factor and a predictor of poor prognosis in breast and lung cancers. Oncogene 29, 5171-5181

12. Wu G, Liu H, He H et al (2014) miR-372 down-regulates the oncogene ATAD2 to influence hepatocellular carcinoma proliferation and metastasis. BMC Cancer 14, $1-11$

13. Wan WN, Zhang YX, Wang XM et al (2014) ATAD2 is highly expressed in ovarian carcinomas and indicates poor prognosis. APJCP 15, 2777-2783

14. Shang P, Meng FL, Liu YD and Chen XW (2015) Overexpression of ANCCAATAD2 in endometrial carcinoma and its correlation with tumor progression and poor prognosis. Tumor Biol 36, 4479-4485

15. Zhang MJ, Zhang CZ, Du WJ, Yang XZ and Chen ZP (2015) ATAD2 is overexpressed in gastric cancer and serves as an independent poor prognostic biomarker. Clin Transl Oncol 18, 1-6

16. Jadvar H, Alavi A, Gambhir SS (2009) [18F]FDG uptake in lung, breast, and colon cancers: molecular biology correlates and disease characterization. J Nucl Med 50, 1820-1827

17. Cho H, Lee YS, Kim J, Chung JY and Kim JH (2013) Overexpression of glucose transporter-1 (GLUT-1) predicts poor prognosis in epithelial ovarian cancer. Cancer Invest 31, 607-615

18. Chae YC and Kim JH (2018) Cancer stem cell metabolism: target for cancer therapy. BMB Rep 51, 319-326

19. Zhuo B, Li Y, Li Z et al (2015) Pl3K/Akt signaling mediated Hexokinase-2 expression inhibits cell apoptosis and promotes tumor growth in pediatric osteosarcoma. Biochem Biophys Res Commun 464, 401-406

20. Wang H, Wang L, Zhang YJ, Wang J, Deng YB and Lin DG (2016) Inhibition of glycolytic enzyme hexokinase II (HK2) suppresses lung tumor growth. Cancer Cell Int 16, 9

21. Boussouar F, Jamshidikia $M$, Morozumi $Y$, Rousseaux $S$ and Khochbin S (2013) Malignant genome reprogramming by ATAD2. Biochim Biophys Acta 1829, 1010-1014

22. Wu K, Wang W, Chen H, Gao W and Yu C (2019) Insulin promotes proliferation of pancreatic ductal epithelial cells by increasing expression of PLK1 through PI3K/AKT and NF-kB pathway. Biochem Biophys Res Commun 509, 925-930

23. Tsai JS, Chao CH and Lin LY (2016) Cadmium activates multiple signaling pathways that coordinately stimulate Akt activity to enhance c-Myc mRNA stability. PLoS One 11, e0147011

24. Hong SY, Yu FX, Luo Y and Hagen T (2016) Oncogenic activation of the PI3K/Akt pathway promotes cellular glucose uptake by downregulating the expression of thioredoxin-interacting protein. Cell Signal 28, 377-383

25. Stine ZE, Walton ZE, Altman BJ, Hsieh AL and Dang CV (2015) MYC, metabolism, and cancer. Cancer Discov 5, 1024-1039

26. Wahlstrom T and Henriksson MA (2015) Impact of MYC in regulation of tumor cell metabolism. Biochim Biophys Acta $1849,563-569$

27. Ciro $M$, Prosperini E, Quarto $M$ et al (2009) ATAD2 is a novel cofactor for MYC, overexpressed and amplified in aggressive tumors. Cancer Res 69, 8491-8498 